\title{
Small scale contributions to the cosmic microwave background: a coherent analysis
}

\author{
M. Douspis ${ }^{1,2}$, N. Aghanim ${ }^{2}$, and M. Langer ${ }^{2}$ \\ 1 LATT, 14 avenue Édouard Belin, 31400 Toulouse, France \\ e-mail: douspis@ast.obs-mip.fr \\ 2 Institut d'Astrophysique Spatiale (IAS), Université Paris-Sud 11, CNRS (UMR 8617), Bâtiment 121, 91405 Orsay, France
}

Received 26 January 2006 / Accepted 22 May 2006

\begin{abstract}
We re-analyse Cosmic Microwave Background data from experiments probing both large and small scales. We assume that measured anisotropies are due not only to primary fluctuations but also, especially at small scales, to secondary effects (namely the SunyaevZel'dovich effect) and possible point source contamination. We first consider primary and secondary anisotropies. For the first time in such analyses, the cosmological dependence of secondary fluctuations is fully taken into account. We show that in that case a higher value of the normalisation $\sigma_{8}$ is preferred, as found by previous studies, but also higher values of the optical depth $\tau$ and power spectrum index $n_{\mathrm{s}}$ are needed. In the second part, we include possible contaminations from unresolved and unremoved point sources. We discuss the effects on the cosmological parameters. We further obtain the best combination of relative contributions of the three kinds of sources to the measured microwave power on small scales at each frequency. Our method allows us to simultaneously obtain cosmological parameters and explain the so-called small scale power excess in a consistent way.
\end{abstract}

Key words. cosmology: cosmic microwave background - cosmology: large scale structure of Universe - cosmology: cosmological parameters

\section{Introduction}

Measurements of the Cosmic Microwave Background (CMB) angular power spectrum are now becoming invaluable observables for cosmology. The detailed shape of this spectrum allows one to determine cosmological parameters with high precision. The CMB anisotropy spectrum has been measured from large to intermediate scales with WMAP (Bennett et al. 2003), Archeops (Benoît et al. 2003) and BOOMERanG (Jones et al. 2005) experiments. At small scales, ACBAR (Kuo et al. 2004), CBI (Pearson et al. 2003; Readhead et al. 2004a), VSA (Rebolo et al. 2004) and BIMA (Dawson et al. 2002) have detected signals in excess of that expected from purely primordial CMB anisotropies. This excess is not fully understood yet but several explanations have been proposed. Some relate to the early Universe (e.g. Cooray \& Melchiorri 2002; Griffiths et al. 2003), some are associated with reionising sources (e.g. Oh et al. 2003) and others are related to the astrophysical processes contributing to the signal at the CMB frequencies (e.g. Bond et al. 2005; Toffolatti et al. 2005). When observing at microwave wavelengths one expects to detect not only primordial CMB fluctuations but also secondary anisotropies as well as galactic and extra-galactic foreground. Astrophysical contributions are, as much as possible, removed during data analysis to produce clean estimates of the CMB angular power spectrum. Nevertheless, residuals may remain and contribute to the measured power.

In order to minimise galactic contamination, most of the small sky coverage experiments (probing small scales) observe at high galactic latitudes in regions where the sky is as much as possible free of galactic emission. Moreover, the galactic signal can be removed through multi-frequency observations, or by masking the contaminated regions. Since most of the residual signal is expected on large scales, galactic emission only very moderately affects small scale CMB observations.

At small angular scales, an additional contribution to the primary CMB signal arises from the interaction of CMB photons with matter along their propagation path. These are the socalled secondary anisotropies (e.g. Hu \& Dodelson 2002, and references therein). The latter are dominated by the thermal Sunyaev-Zel'dovich (SZ) effect (Sunyaev \& Zel'dovich 1972, 1980), i.e. CMB photon inverse Compton scattering off free electrons in the hot intra-cluster medium. When clusters are known and resolved by an instrument, they can easily be removed from CMB maps. However, not all clusters are known and we expect a contribution to the microwave signal from unresolved/unknown clusters. The SZ effect being frequency dependent (contrary to primary CMB fluctuations) and its spectral signature being known, one can use this information to detect and remove the SZ signal from unknown clusters in a map if multi-frequency observations are conducted. On the contrary, with single frequency experiments, we are left with an SZ contamination at small scales.

Contamination by extra-galactic sources has long been studied for $\mathrm{CMB}$ and SZ observations. The effects of radio sources were investigated for low frequency experiments (e.g. Ledlow \& Owen 1996; Cooray et al. 1998; Toffolatti et al. 1998; Sokasian et al. 2001; de Zotti et al. 2005; González-Nuevo et al. 2005). At high frequencies dusty galaxies emitting in the infra-red are the dominant sources of contamination (e.g. Blain 1998; White \& Majumdar 2004; Lagache et al. 2005). Contamination by point sources can be monitored by interferometric arrays, and multifrequency observations should help us in reducing the additional signal through component separation. Once again, however, a 
residual contamination is likely to remain, especially in view of the uncertainties in the modelling of the sources themselves.

The excess power reported by small scale CMB experiments may thus be due to several astrophysical signals expected to contribute at those scales. In order to correctly estimate the relative and possible contribution of the secondary effects or extragalactic signal on top of the primary CMB anisotropy, one needs a coherent analysis. Cosmological dependences are expected for the CMB and SZ effect, and SZ and point sources contribute on similar scales. Thus, unlike previous studies (e.g. Goldstein et al. 2003; Bond et al. 2005), we do not decorrelate small scales from those on large scales in our analysis of the CMB data. On the contrary, we look for the best model that fits data simultaneously on large and small scales. Hence, assuming that primary, secondary and point source signals all contribute to the model, we present a coherent analysis (i.e. including the full cosmological dependence of secondary effects) of the CMB angular power spectrum as measured from large $(\ell=2)$ to small $(\ell=10000)$ scales.

In the next two sections we describe the CMB data we used and our modelling of the power spectra of the different contributions that enter the analysis. We then present our results in terms of cosmological parameters inferred from an analysis coherently accounting for primary, secondary and extra-galactic contributions. We show in each case the relative contributions of all considered signals, and discuss our results.

\section{Observations}

CMB temperature anisotropies have been measured over a wide range of scales with high significance. WMAP has yielded the best measurements from large to intermediate angular scales, and together with Archeops and BOOMERanG, offers an estimate of a series of features (peaks and troughs) consistent with those expected from acoustic oscillations in the primordial fluid. ACBAR, CBI, VSA and BIMA observed smaller scales, those affected by exponential damping of power of primary fluctuations due to photon diffusion and to the finite thickness of the surface of last scattering. The CMB polarisation (TE, EE, BB) has also been measured by several experiments (DASI, Leitch et al. 2005; BOOMERanG, Piacentini et al. 2005; Montroy et al. 2005; CBI, Readhead et al. 2004b; WMAP, Kogut et al. 2003). Altogether, CMB temperature and polarisation anisotropy measurements now enable us to constrain cosmological parameters and hence the underlying theoretical models. A firm detection of the first peaks in the CMB anisotropy angular power spectrum and a damping at smaller scales has been obtained. However, an excess of power at scales around a few arcminutes $(\ell>2000)$ has been found in the data (see Sect. 1).

In the present study, we consider only a subset of data that allows us to explore the whole range of measured scales. We use WMAP and BOOMERanG data, whose major advantage is an accurate measurement of the primordial signal at $\ell<1500$, and that of ACBAR, BIMA and CBI (at higher $\ell$ ) that exhibit an excess of power with respect to the expected primary contribution. In addition, we take into account in our analysis the polarised data from WMAP and BOOMERanG. Altogether, this sample represents 1470 data points.

\section{Contributions to the signal}

Observations of the sky may contain many astrophysical processes which contribute to the signal along the line of sight. They

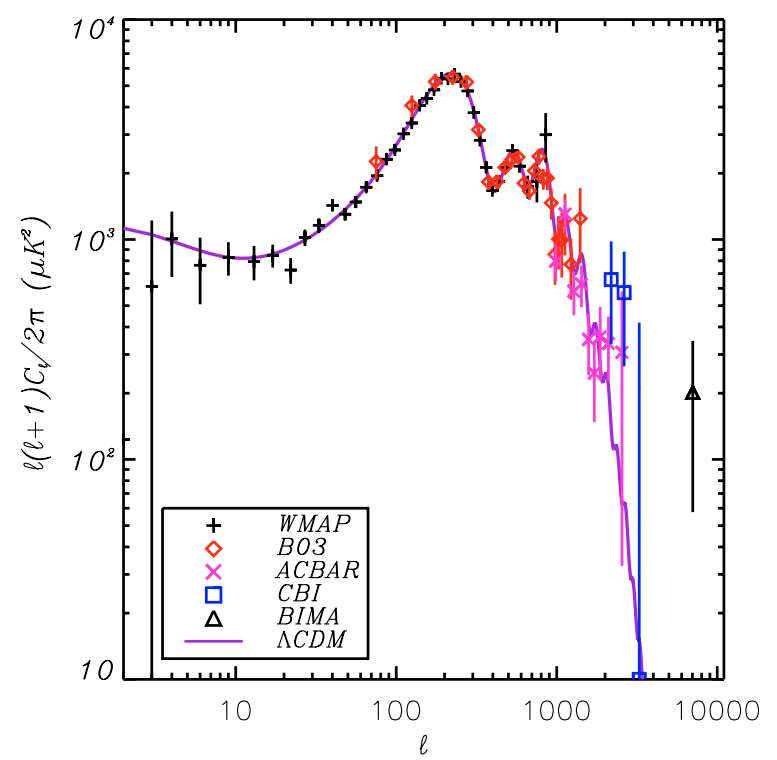

Fig. 1. Data used in the analysis. Overplotted is the best model when only primary fluctuations are considered and fitted to data with $\ell<1500$ (purple solid line).

have to be either accounted for or removed before analysing the signal of interest. Usually CMB experiments observe high latitude patches of the sky from which galactic contaminations are subtracted. In the following, we will therefore neglect potential galactic residuals and focus exclusively on the extra-galactic contributions described below.

\subsection{Primary fluctuations}

We call primary fluctuations those anisotropies that are present at recombination. They may be due to intrinsic temperature fluctuations, density fluctuations or velocity fluctuations in the primordial plasma. The derived CMB angular power spectrum shows a particular shape, the main characteristics of which are independent of the cosmology: a plateau at large scales, a succession of peaks and a damping at small scales. The details of those features are, on the contrary, highly dependent on the cosmological parameters. The position of the first acoustic peak is sensitive for example to the total density of the Universe. The CMB power spectrum is now well used to determine cosmological parameters by fitting the data of Fig. 1 with theoretical predictions. Here, the temperature and polarisation angular power spectra for primary fluctuations were computed using the Boltzmann code CAMB (Lewis et al. 2000).

\subsection{Secondary fluctuations - SZ effect}

The Sunyaev-Zel'dovich (SZ) effect is the dominant secondary effect at scales between a few tens arcminutes to a fraction of an arcminute. It has now been observed in the direction of a few tens of known clusters (e.g. Carlstrom et al. 2002, and references therein). The SZ effect has a particular spectral signature which induces an excess of brightness at high frequency and a deficit of brightness at low frequency in the Rayleigh-Jeans regime. This makes it in theory easily distinguishable from other contributions to the millimetre (mm) and sub-millimetre (submm) sky when multi-frequency observations are conducted. When the sky is observed at one single frequency (e.g. CBI, VSA, BIMA, AMIBA Lo et al. 2001) it is a priori impossible to clean the 
signal from SZ contamination except in the direction of known clusters. An SZ contribution is thus expected at small angular scales in CMB surveys. It is usually estimated through the SZ angular power spectrum which can be inferred directly from cosmological numerical simulations or computed analytically (e.g. Komatsu \& Seljak 2002, for a review, and references therein).

In the present study, we choose to compute the SZ power spectrum using an analytic approach. Such a choice is particularly important for a coherent analysis of the CMB anisotropies including primary and secondary signals. Indeed, the SZ power spectrum depends on the cosmological parameters through the number of SZ sources and their characteristics (gas content, temperature, evolution...). Accounting for modifications through numerical simulations requires numerous simulations with different parameters which is both time and storage consuming. Numerical simulations are also limited by resolution and size. This affects the estimates of contributions at small and large angular scales. The dependence on cosmological parameters is more directly and easily handled with analytic computations (see for example Komatsu \& Seljak 2002). In practise, we have computed the power spectrum of sources with masses between $M_{\text {min }}=5 \times 10^{11} h^{-1}$ and $M_{\max }=5 \times 10^{15} h^{-1}$ solar masses up to $z=7$ following Komatsu \& Seljak (2002). The Poisson contribution to the $\mathrm{SZ}$ power spectrum $C_{\ell}^{\mathrm{SZ}}$ is given by:

$C_{\ell}^{\mathrm{SZ}}=g(v)^{2} \int_{0}^{z_{\max }} \frac{\mathrm{d} V(z)}{\mathrm{d} z} \mathrm{~d} z \int_{M_{\min }}^{M_{\max }} \frac{\mathrm{d} N(M, z)}{\mathrm{d} M}\left[\hat{y}_{\ell}(M, z)\right]^{2} \mathrm{~d} M$

where $V(z)$ is the comoving volume element, $\mathrm{d} N(M, z) / \mathrm{d} M$ is the comoving mass function and $\hat{y}_{\ell}(M, z)$ is the 2D Fourier transform of the projected Compton parameter (the line of sight integral of the intra-cluster gas pressure) which characterises the amplitude of the SZ effect. The spectral dependence is given by $g(v)$. We used a modified Press-Schechter mass function (Sheth \& Tormen 1999) and accounted for possible variations of the spectral index $n_{\mathrm{s}}$ of the initial density power spectrum through the "degenerated shape parameter" defined in Wu (2001). For the individual contribution $\hat{y}_{\ell}(M, z)$, we followed the description in Komatsu \& Seljak (2002) of a hot gas in hydrostatic equilibrium in a universal dark matter potential with a constant polytropic equation of state. Detailed effects of the gas profile and mass function on the SZ power spectrum are given in Komatsu \& Seljak (2002) to which we refer the reader. Using Eq. (1) we can compute the expected signal from a population of SZ sources for any given set of cosmological parameters, at each multipole $\ell$ and for any observing frequency $v$. The SZ power spectrum has a bell-like shape, the amplitude of which depends mainly on the normalisation parameter $\sigma_{8}$, but also on the mass and redshift ranges together with the set of physical and cosmological parameters used in the computation. A correlated contribution to Eq. (1) would modify the low $\ell$ part of the spectrum by adding power at large angular scales. The relative contribution of SZ anisotropies at those scales being small we choose not to take the correlations into account. The EE and BB polarisation power spectra induced by clusters are orders of magnitude smaller than the primary polarised power spectra (Liu et al. 2005). We therefore do not take these into account in the present analysis.

\subsection{Point source contamination}

The extra-galactic contaminations depend on the observing frequency through the spectral energy distribution of the sources and their number counts. The extra-galactic contributions to the CMB signal are usually classified broadly into radio galaxies emitting mainly at low frequencies and dusty IR galaxies at high frequencies with a crossing point at about $150 \mathrm{GHz}$ where both contributions are of the same order (Aghanim et al. 2005).

Star forming galaxies emit, in the near-IR, optical and UV domains and also in the far-IR (up to $90 \%$ of their radiation) and submm, an emission associated with the radiation absorbed and re-emitted by dust. The total emission from unresolved and faint so-called IR galaxies is responsible for the Cosmic Infra-red Background, the fluctuations of which have been detected (Lagache et al. 2000; Matsuhara et al. 2000; Miville-Deschênes et al. 2002; Kiss et al. 2001). This is an important contaminant for SZ and CMB measurements at high frequencies $(\geq 100 \mathrm{GHz})$.

Radio galaxy emission is dominated by synchrotron emission from relativistic electrons spiralling around magnetic field lines. The synchrotron spectrum generally follows a power law $v^{\alpha}$, with $-1<\alpha<-0.5$. However, those spectra need not be simple power laws (Herbig \& Readhead 1992) but may have curved shapes; they even can exhibit inverted slopes (with $\alpha>0$ ); moreover some of the radio sources may be variable. Radio sources are rather well catalogued at low frequencies thanks to NVSS (Condon et al. 1998) and FIRST (White et al. 1997) surveys. The all sky survey from WMAP observations at $41 \mathrm{GHz}$ (Bennett et al. 2003) has provided us with information at higher frequencies where the radio sources are poorly known. In order to assess the level of contamination due to radio sources at the frequencies of interest for the CMB (between 30 and $200 \mathrm{GHz}$ ), studies were conducted and models proposed to predict radio source counts (Toffolatti et al. 1998; Sokasian et al. 2001; de Zotti et al. 2005). In particular, the model by de Zotti et al. (2005) found to be in quite a good agreement with detections of radio sources by WMAP provides us with good estimates of the radio source contribution to the CMB signal.

Point source contamination can be reduced in, or removed from, CMB observations. This is usually done using multifrequency data and component separation techniques, or using monitored observations at the same frequency as CMB experiments, or using spatial correlations with already existing source catalogues. Different strategies for point source cleaning were used by the groups of ACBAR, BIMA and CBI, and are described in detail in the articles by Dawson et al. (2002); Mason et al. (2003); Kuo et al. (2004) and Readhead et al. (2004a). These strategies comprise: pointed observations at the CMB frequencies of sources at lower frequencies, direct counts using deep and mosaic images at the observing frequency, removal of faint sources from external catalogues, ancillary surveys at lower frequencies, using existing catalogues... However, the uncertainties in the physical description of the contaminating emissions (extrapolations from frequency to frequency, source variability...) together with the sensitivities of the complementary data leave an unremoved contamination that is likely to contribute to the signal. Toffolatti et al. (2005) have recently revisited the contributions of extra-galactic point sources to the arcminute scale measurements of ACBAR, BIMA and CBI at the relevant frequencies, namely $28.5,30$ and $150 \mathrm{GHz}$. In particular using predictions by de Zotti et al. (2005) updating the source model of Toffolatti et al. (1998), they have shown that faint sources with fluxes too weak to be detected in ancillary surveys might contribute a significant fraction of the arcminute signal.

For our study, we consider that such an unremoved component does indeed contribute to the measured arcminute signal. We do not take into account possible enhancement by lensing. We model the point source contribution in a very simple way, assuming it is well described by a power spectrum $C_{\ell}^{\mathrm{PS}}=\alpha_{v_{\mathrm{obs}}}^{\mathrm{PS}} \ell^{2}$, 
typical of a Poisson distribution. The parameter $\alpha_{v_{\mathrm{obs}}}^{\mathrm{PS}}$ accounts for the rms contribution of unremoved point sources (i.e. below the detection limit or unaccounted for) at a given observing frequency $v_{\text {obs }}$. As a consequence, a different power spectrum, i.e. a different $\alpha_{v_{\mathrm{obs}}}^{\mathrm{PS}}$ is assumed for each experiment (see Sect. 4). Correlations between sources modify the contribution at large angular scales by adding power to the shot noise and thus they are expected to change the slope of the associated power spectrum (e.g. Song et al. 2003; Negrello et al. 2005). However, in view of the still limited knowledge we have of it, we choose not to take the source correlation into account.

Point sources may in principle also contribute to the polarised signal on small scales. Predictions of CMB polarisation level from extra-galactic radio sources are summarised by Tucci et al. (2005), who show that radio galaxy contribution is largely dominated by synchrotron emission from our galaxy for all relevant frequencies. Similarly, dusty IR galaxies are also expected to contribute to the polarised signal. Not much data is available on IR galaxy polarisation, and firm predictions of its contribution to the CMB are difficult to make. Assuming that the level of dust polarised emission in external galaxies is not higher than that of the Milky Way, it has been shown (Tucci et al. 2005) that polarisation from IR galaxies is sub-dominant at frequencies below $1000 \mathrm{GHz}$ and for $\ell<3000$. We therefore neglect polarisation from radio and IR galaxies in our analysis.

\section{Analysis}

\subsection{Method}

In this work, we account for the contributions to the CMB signal of the different astrophysical sources (primary fluctuations, secondary anisotropies and residual extra-galactic point sources) in a coherent way. We compute their associated power spectra by taking into account the dependences on the cosmological parameters of each signal. Note however that our empirical modelling of residual point sources does not explicitly show such a dependence. We analyse the CMB data shown in Fig. 1 and look for the set of best cosmological parameters (best fit model) and corresponding error bars when assuming that one, two or three of the contributions described in Sect. 3 contribute to the measured signal.

As seen in the previous section, the SZ angular power spectrum is frequency dependent. We thus consider three observing frequencies 150,30 and $28.5 \mathrm{GHz}$. The level of contamination from residual point sources is related to the beam size of each experiment, to its sensitivity and to the flux limit which accounts also for possible source extraction using cross-correlations with other wavelengths. Therefore, the point source contribution in terms of power spectrum is fitted, in the following, independently for each experiment. As a result, the total power spectrum (sum of primary, secondary and unremoved point sources) is also frequency dependent and thus fitted for each observing frequency, i.e. for each small scale experiment (ACBAR, BIMA, $\mathrm{CBI}$ ) added to WMAP and BOOMERanG.

We use a Monte-Carlo Markov Chain approach allowing us to account for a large number of parameters without using intensive computational resources. We apply the test of convergence developed by Dunkley et al. (2005) to check the robustness of our results. The likelihood of WMAP data is computed by using the WMAP collaboration routines ${ }^{1}$ whereas the likelihoods

\footnotetext{
1 These routines are available at http://lambda.gsfc.nasa.gov/product/map/current/ m_sw.cfm
}

of the other data (ACBAR, BIMA, CBI) are computed with the Bond, Jaffe \& Knox approximation (Bond et al. 2000) when the necessary information is provided (Gaussian-like approximation otherwise). When all the astrophysical contributions are taken into account the final likelihood $\mathcal{L}$ is:

$\mathcal{L}=\prod_{i}^{N} \mathcal{L}_{i}\left(C_{\ell}\left(i, v_{i}, \theta\right) \mid C_{\ell}^{\mathrm{obs}}(i)\right)$

where $C_{\ell}^{\mathrm{obs}}(i)$ is the angular power spectrum of an experiment $i$ observing at frequency $v_{i}, \theta$ is the set of cosmological parameters and the total theoretical angular power spectrum is:

$C_{\ell}\left(i, v_{i}, \theta\right)=C_{\ell}^{\text {primary }}(\theta)+C_{\ell}^{\mathrm{SZ}}\left(v_{i}, \theta\right)+C_{\ell}^{\mathrm{PS}}\left(v_{i}\right)$,

with $C_{\ell}^{\mathrm{PS}}\left(v_{i}\right)=\alpha_{v_{\mathrm{obs}}}^{\mathrm{PS}} \ell^{2}$. Note that in the case of WMAP and BOOMERanG experiments, the $C_{\ell}^{\text {primary }}(\theta)$ (and a fortiori the likelihood $\mathcal{L}_{i}$ ) includes the polarisation signal. We consider throughout a flat cosmological model without contribution from neutrinos or tensor modes. We assume a power law initial power spectrum for the density perturbations with spectral index $n_{\mathrm{s}}$. The remaining cosmological parameters are: the baryon budget, $\Omega_{\mathrm{b}} h^{2}$, the cold dark matter content, $\Omega_{\mathrm{cdm}} h^{2}$, the Hubble constant, $H_{0}$, the normalisation factor $\sigma_{8}$ (given by the amplitude of fluctuation on a sphere of $8 h^{-1} \mathrm{Mpc}$ ), and the Thomson scattering optical depth $\tau$ (related to the reionisation history of the Universe), to which we add the amplitudes, $\alpha_{v_{i}}^{\mathrm{PS}}$, of the point source contributions for each experiment.

\subsection{Results}

In the following we consider first the case where primary and secondary anisotropies are present, then we add the contribution of unremoved point sources. We show in both cases the set of derived cosmological parameters and point out the effects of an additional, non primary contribution to the signal.

\subsection{1. $\mathrm{CMB}+\mathrm{SZ}$ case}

Atmospheric, galactic and resolved point sources are usually well removed from CMB observations; the SZ effect thus has first been advocated to explain the excess of power detected at small scales. Previous analyses often decorrelate anisotropies at large scales, associated with the primary signal, from those at small scales related to the secondary signal. A primary power spectrum is thus generally fitted with data at $\ell<1500$. It is added to a template SZ power spectrum (generally obtained from numerical simulations with fixed cosmological parameters). In such analyses, the amplitude of the SZ power spectrum is the only parameter set free (often labelled $\sigma_{8}^{\mathrm{SZ}}$ and related to the normalisation $\sigma_{8}$ ). Such a template spectrum is then fitted to the data available above $\ell=1500$ and the SZ contribution, and therefore $\sigma_{8}^{\mathrm{SZ}}$, are derived.

As seen in Sect. 3, the SZ angular power spectrum depends on the cosmological parameters through the volume element, the number density and the individual Compton parameter. The relative contribution of primary and secondary signals are thus strongly connected and cannot be formally separated. As a result, although the signal from primary fluctuations is weak at small scales $(\ell>1500)$ it is incorrect to decorrelate the two contributions in the fit.

In the present study, we thus fit all data described in Sect. 2 by angular power spectra summing the primary and secondary contributions, with free cosmological parameters. The results of 

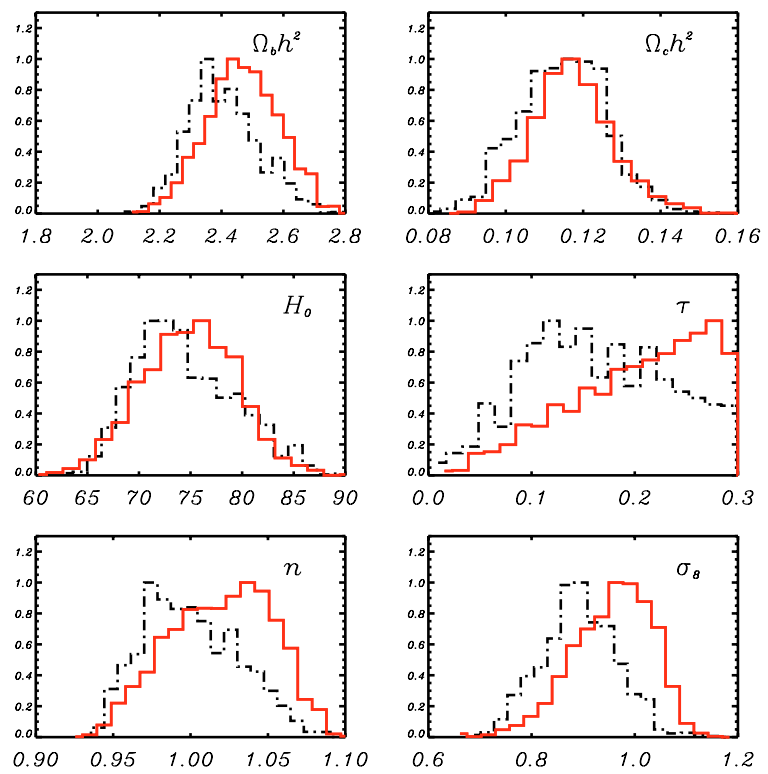

Fig. 2. Marginalised one-dimensional distributions for the cosmological parameters investigated. The black dotted-dashed line shows the curves for the case where data with $\ell<1500$ are fitted with pure CMB models. The red solid lines are derived from the MCMC run with all data and SZ contribution added to CMB (Run 1). The value of $\Omega_{\mathrm{b}} h^{2}$ as been multiplied by 100 for clarity.

the corresponding MCMC run (Run 1) are shown in terms of one dimensional likelihood functions (solid red lines) in Fig. 2. The latter are compared with the likelihood functions obtained by fitting $\ell<1500$ data by primary CMB power spectra only (black dotted-dashed lines).

The main effect of coherently adding the SZ signal is to allow the cosmological models to fit the small scale data. This implies that high values of $\sigma_{8}$ (median value: 0.97 ) and $n_{\mathrm{s}}$ (median value: 1.02 ) are preferred. Both parameters tend to amplify the power at small scales. In particular, as seen before, the amplitude of the SZ power spectrum depends on $\sigma_{8}$. The higher $\sigma_{8}$ the larger the SZ signal. Such an effect was recognised in previous studies (Goldstein et al. 2003; Bond et al. 2005). A coherent analysis taking into account both primary and SZ signals allows us to emphasize the effects of a small scale excess of power on the other cosmological parameters. As a matter of fact, in order to compensate for the additional small scale power and keep the large scale power at WMAP amplitude, large values of the optical depth $\tau$ (close to 0.3 ) are necessary to balance the effects of $\sigma_{8}$ and $n_{\mathrm{s}}$. Such values are allowed by the WMAP TE power spectrum at large scales (see Kogut et al. 2003; Melchiorri et al. 2005). This is the well-known degeneracy between $\sigma_{8}$ or $n_{\mathrm{s}}$ and $\tau$ illustrated in Fig. 3. The other cosmological parameters $\Omega_{\mathrm{b}} h^{2}$, $\Omega_{\mathrm{m}} h^{2}$ and $H_{0}$ are only very marginally affected by the excess of power at small scales, but our results seem to suggest that slightly higher values of $\Omega_{\mathrm{m}} h^{2}$ and $H_{0}$ are favoured.

The best model, defined as the one with cosmological parameters being the median of the distributions (Fig. 2), is shown in Fig. 4. The corresponding cosmological parameters are: $\Omega_{\mathrm{b}} h^{2}=$ $0.025, \Omega_{\mathrm{cdm}} h^{2}=0.116, H_{0}=75.33 \mathrm{~km} \mathrm{~s}^{-1} \mathrm{Mpc}^{-1}, \tau=0.22$, $n=1.02, \sigma_{8}=0.97$. This model slightly improves the goodness of fit as compared to the best pure primary spectrum without adding any other parameter. In Fig. 4, the corresponding total angular power spectrum is plotted in black solid line and the primary and secondary contributions appear respectively in purple dotted-dashed line and orange long-dashed line. The difference

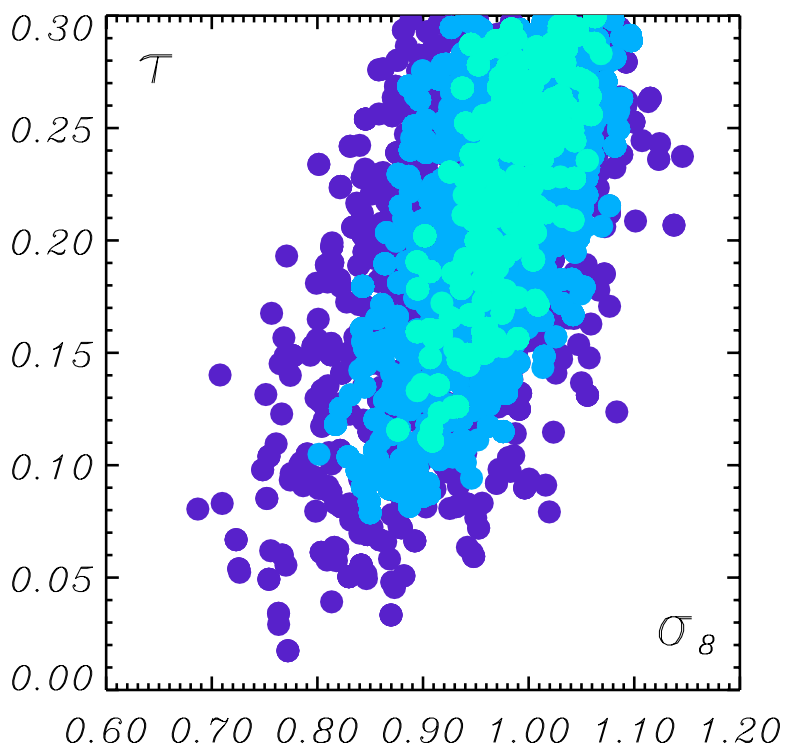

Fig. 3. Two-dimensional plot of the degeneracy between $\sigma_{8}$ and $\tau$ obtained from Run 1. Coloured shaded regions mark the $1,2,3 \sigma$ contours from light to dark.

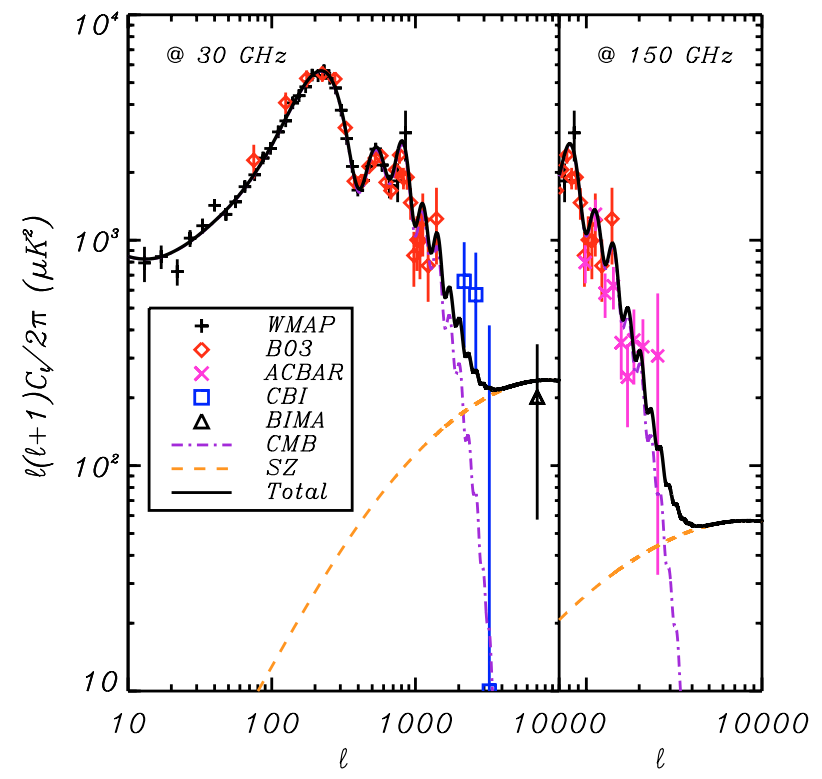

Fig. 4. Total power spectrum (in solid black line) overplotted on the data for the two frequencies considered. The SZ power spectrum amplitudes are function of the frequency range considered (magenta dashed lines) while the primary (purple dot-dashed line) contribution is not.

in SZ amplitude between the two plots at 30 and $150 \mathrm{GHz}$ is due to the frequency dependence $g(v)^{2}$ in Eq. (1). The relative contributions to the total power spectrum are shown as functions of $\ell$ in Fig. 5; the primary contribution is displayed in white and the secondary contribution is displayed in orange/grey. This figure gives the contribution of the SZ signal observed in microwave in a joint and coherent analysis where cosmological parameters are adjusted simultaneously for both primary and secondary anisotropies to fit current data. At $\ell=3000$ the $\mathrm{CMB}$ signal is dominated by the SZ contribution which represents $86 \%$ and $59 \%$, at 30 and $150 \mathrm{GHz}$ respectively, of the total signal. As one can see in Fig. 2, the best model that fits $\ell<1500$ data, free from SZ contribution, is still a good model for the whole data set (up to $\ell=10000$ ). The $\mathrm{SZ}$ contribution at 30 (150) $\mathrm{GHz}$ 


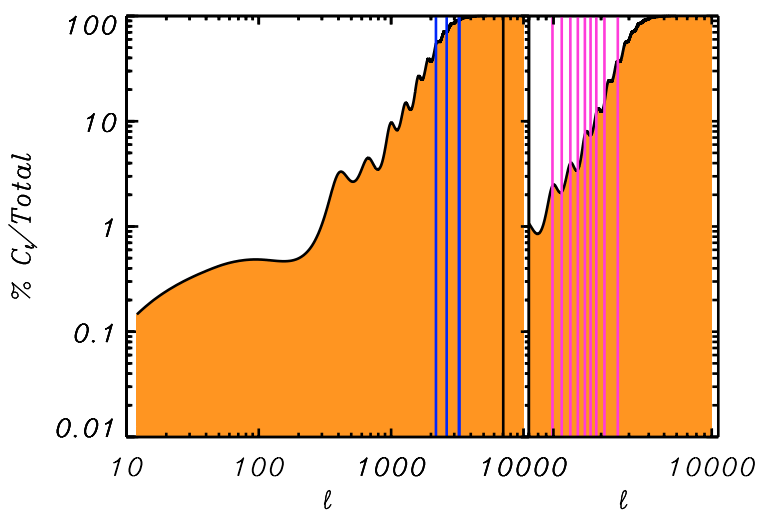

Fig. 5. Contributions of primary and SZ power spectra to the total spectrum (solid black line of Fig. 4). The orange shaded (white) region gives the percentage of total power due to SZ (resp. primary fluctuations) contribution.

at $\ell=3000$ varies from 0 to $98 \%$ (91\%) for the models within the $68 \%$ confidence interval in the 6 dimensional cosmological parameter space. The actual data set at small scales is clearly poorly constraining due to the large error bars and the intercalibration problem. The situation should improve with future observations. Note that the present analysis does not account for the statistical dispersion which induces a large dispersion in the $\mathrm{SZ}$ contribution. Most of the SZ signal is due to rare massive clusters. As noticed in Fig. 2 of Cooray \& Melchiorri (2002), the SZ variance is expected to be much larger due to the nonGaussian nature of the SZ signal than that of a Gaussian distribution. Multi-frequency observations of the same field are the only way to resolve this problem.

\subsection{2. $\mathrm{CMB}+\mathrm{SZ}+\mathrm{PS}$ case}

As seen in Fig. 4, the SZ angular power spectrum does not fully explain the excess power of some data points at small scales. Of course, the latter could be $1 \sigma$ events. Nevertheless, as discussed above, some contribution from unresolved and unremoved point sources is also expected in the small scale signal. We want to quantify the effects of this additional contribution. We thus repeat our analysis by including the point sources in addition to primary and SZ contributions (MCMC Run 2). Modelling the angular power spectrum by $\ell(\ell+1) C_{\ell} / 2 / \pi \equiv \alpha_{v_{i}}^{\mathrm{PS}}(\ell / 2000)^{2}$, we introduce 3 new parameters $\alpha_{v_{i}}^{\mathrm{PS}}$ noted $A, B, C$ and describing the amplitudes of point sources remaining in each experiment, ACBAR, BIMA and CBI respectively. These new parameters give the amplitude in $\mu \mathrm{K}^{2}$ of the point source contribution at $\ell=2000$.

The output of Run 2 is shown in Fig. 6 in terms of one dimensional likelihood functions. We notice in this case that the estimated cosmological parameters (red/light lines) are all in perfect agreement with the results obtained by considering $\ell<1500$ data and primary fluctuations only (black/dark lines). The addition of an unremoved point source contribution at small scales improves the goodness of fit more than when adding SZ only. The values of cosmological parameters $\Omega_{\mathrm{b}} h^{2}, \Omega_{\mathrm{m}} h^{2}$ and $H_{0}$ are almost identical to those obtained with primary CMB only and $\ell<1500$ data. The parameters $\tau, n_{\mathrm{s}}$ and $\sigma_{8}$ also converge to values obtained in pure primary CMB analyses. In the present case (primary CMB, SZ effect and unresolved point sources) we find a perfect agreement between large and small scale data converging towards the following median values for
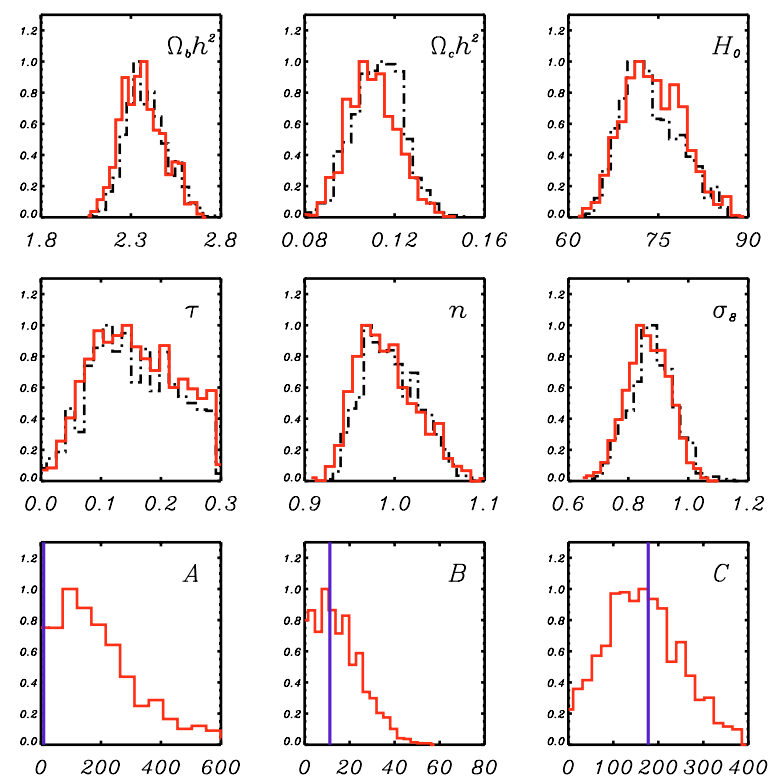

Fig. 6. One-dimensional distribution of cosmological and point sources amplitude parameters from MCMC Run 2. The vertical (blue) lines in the three lower panels give the amplitude of point source contributions as computed by Toffolatti et al. (see text).

the cosmological parameters: $\Omega_{\mathrm{b}} h^{2}=0.0237, \Omega_{\mathrm{cdm}} h^{2}=0.111$, $H_{0}=74.6 \mathrm{~km} \mathrm{~s}^{-1} \mathrm{Mpc}^{-1}, \tau=0.16, n=0.99, \sigma_{8}=0.87$.

In addition to the cosmological parameters, we obtain estimations of the point source amplitudes for each experiment. The corresponding values are $181,14,170 \mu \mathrm{K}^{2}$ for ACBAR, BIMA, CBI respectively. A recent study based on a physical model for point sources in radio and infra-red domains (Toffolatti et al. 2005) has shown that the excess of power at small angular scales could be explained almost entirely by the contribution from unresolved point sources. We compare the predictions of Toffolatti et al. (2005) to our results given by the one dimensional likelihood functions of $A, B$ and $C$ in Fig. 6. We find that predictions from Toffolatti et al. are not only in agreement with our results but their values correspond to the maxima of the distributions in Fig. 6.

We plot the contributions of each component to the signal in Fig. 7 for our best fit model. The relative contributions of the three astrophysical processes to the total signal are summarised in Fig. 8. The primary CMB is displayed in white, the SZ effect is in orange/light grey and the point sources are in red/dark grey. At $\ell=3000$, the relative contributions are 2, 40 and $9 \%$ for SZ contributions at (ACBAR, BIMA, CBI frequencies) and 91, 32 and $85 \%$ for the point source signals (at the same frequencies). Again, these numbers, corresponding to the best model, have to be taken with caution as they do not account for the larger statistical dispersions due to the non-Gaussian nature of both SZ and point sources. Furthermore when the $68 \%$ confidence interval in 6 dimensions is considered, the SZ (PS) contributions at $\ell=3000$ vary from 0 to $80 \%, 0$ to $96 \%$ and 0 to $91 \%$ (0 to $99 \%, 0$ to $85 \%$ and 0 to $98 \%$ ) for respectively ACBAR, BIMA and $\mathrm{CBI}$.

\section{Discussion}

As we have seen in Sect. 4.2.1, the excess power on small scales could be attributed to the sole contribution of SZ sources (86\% and $59 \%$ at 30 and $150 \mathrm{GHz}$ at $\ell=3000$ ). In that case, high values of $\sigma_{8}, n_{\mathrm{s}}$ and of the Thomson scattering optical 


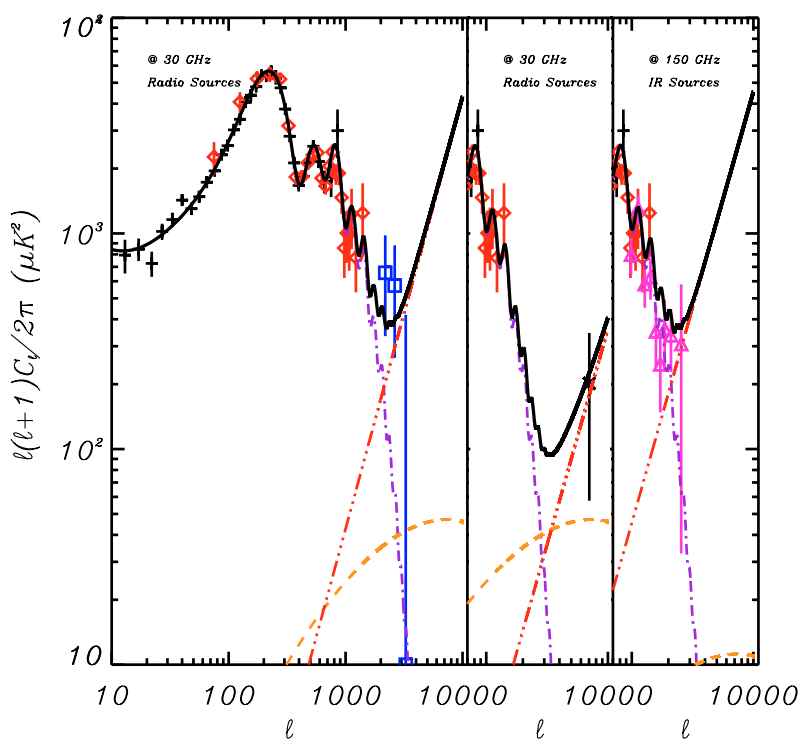

Fig. 7. Best model using all contributions. Primary in purple, SZ in orange, point sources in red.

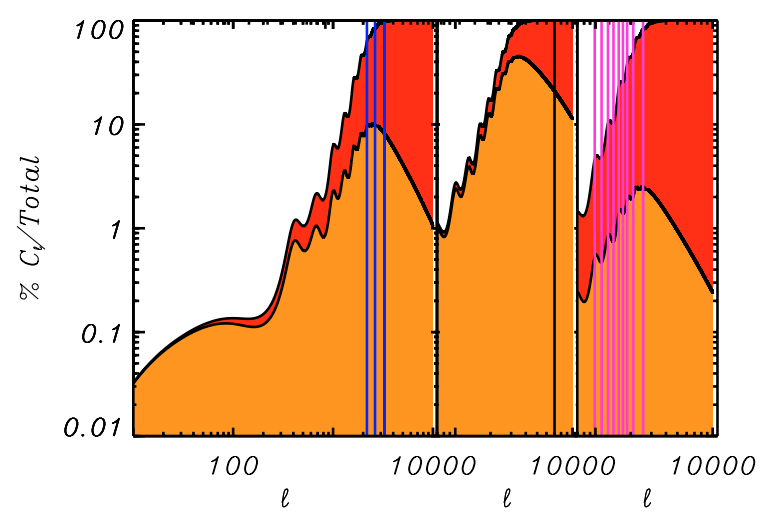

Fig. 8. Relative contributions of primary $\mathrm{CMB}, \mathrm{SZ}$ effect and unremoved point sources power spectra. Primary CMB is displayed in white, $\mathrm{SZ}$ effect is in orange/light grey and point sources are in red/dark grey. From left to right, the vertical lines mark the $\ell$ s observed by CBI, BIMA, ACBAR experiments.

depth $\tau$ are favoured. This in turn may suggest that the Universe went through a very extended reionisation period, which remains compatible with present observational constraints as reported by Melchiorri et al. (2005). However, once we include the possibility of an unremoved or unresolved point source contribution, the likelihood functions of $\tau$ and $\sigma_{8}$ that we obtain peak roughly at the same values as obtained with $\ell<1500$ primary CMB data only.

Our method parametrising the extra-galactic source power spectrum through the parameters $A, B, C$ enables us to probe the possible contributions to the signal. Furthermore, it allows us to test the consistency of specific PS physical models with the cosmological model. In the case of a mismatch, our method would imply the need for different physical descriptions of the source (number counts, evolution, spectral energy distribution).

A substantial fraction of point source contribution seems allowed (see Fig. 8 and Sect. 4.2.2). Such a contribution is in agreement with extra-galactic number count predictions (Toffolatti et al. 2005). We noted however that statistical dispersion due to the non-Gaussian nature of SZ and point sources is likely to significantly reduce the actual contributions. In addition given the current data, large ranges of PS contribution are allowed. From the CMB standpoint, despite the excellent agreement of Toffolatti's model, on the one hand, the SZ effect could effectively contribute and solely explain the excess of power at small scales. On the other hand, different point source models are allowed by current data.

Despite their quality, current data are still not constraining enough for such multi-contribution studies. In addition to their intrinsic error bars, the use of multiple experiments introduces additional inter-calibration uncertainties. Ideally, we would need an experiment that would observe continuously from $\ell=2$ to $\sim 10000$. The Planck satellite will only observe up to $\ell \sim 2000$. However, even then we will still lack data at high and intermediate multipoles, especially in the range $\ell \in[2000,10000]$. In a few years from now, a combination of Planck and future ground based high resolution experiments will help in reducing the error bars and in dealing with the inter-calibration problem.

\section{Conclusions}

We presented the first coherent analysis of CMB data available from $\ell=2$ to $\ell=10000$, simultaneously taking into account contributions from the primary fluctuations and secondary anisotropies as well as point source contamination. We have explicitely taken into account in a consistent way the cosmological dependence of secondary anisotropies. In the first part of our work, this allowed us to confirm that the SZ effect contribution to the CMB power spectrum could explain the so-called small scale power excess, as found in previous studies. We showed however that this requires not only high values of $\sigma_{8}$, but also high values of the spectral index $n_{\mathrm{s}}$ and of the Thomson scattering optical depth $\tau$, leaving other cosmological parameters mostly unaffected.

In the second part of our work, we also included unresolved/unremoved point source contamination. In that case, we recover the cosmological parameter values as determined from large-scale CMB data only. Our consistent analysis introduces a parametrisation of the point source signal. Consequently, we obtain estimates of the contribution of each CMB source to the total power spectrum. Our method hence allows us to test for cosmological consistency of physical models of point source populations.

Acknowledgements. The authors thank Simon Prunet for discussions. MD acknowledges financial support from CNES.

\section{References}

Aghanim, N., Hansen, S. H., \& Lagache, G. 2005, A\&A, 439, 901 Bennett, C. L., Halpern, M., Hinshaw, G., et al. 2003, ApJS, 148, 1 Benoît, A., Ade, P., Amblard, A., et al. 2003, A\&A, 399, L25 Blain, A. W. 1998, MNRAS, 297, 502

Bond, J. R., Contaldi, C. R., Pen, U.-L., et al. 2005, ApJ, 626, 12 Bond, J. R., Jaffe, A. H., \& Knox, L. 2000, ApJ, 533, 19

Carlstrom, J. E., Holder, G. P., \& Reese, E. D. 2002, ARA\&A, 40, 643

Condon, J. J., Cotton, W. D., Greisen, E. W., et al. 1998, AJ, 115, 1693

Cooray, A., \& Melchiorri, A. 2002, Phys. Rev. D, 66, 3001

Cooray, A. R., Grego, L., Holzapfel, W. L., Joy, M., \& Carlstrom, J. E. 1998, AJ, 115, 1388

Dawson, K. S., Holzapfel, W. L., Carlstrom, J. E., et al. 2002, ApJ, 581, 86

de Zotti, G., Ricci, R., Mesa, D., et al. 2005, A\&A, 431, 893

Dunkley, J., Bucher, M., Ferreira, P. G., Moodley, K., \& Skordis, C. 2005, MNRAS, 356, 925

Goldstein, J. H., Ade, P. A. R., Bock, J. J., et al. 2003, ApJ, 599, 773

González-Nuevo, J., Toffolatti, L., \& Argüeso, F. 2005, ApJ, 621, 1

Griffiths, L. M., Kunz, M., \& Silk, J. 2003, MNRAS, 339, 680

Herbig, T., \& Readhead, A. C. S. 1992, ApJS, 81, 83 
Hu, W., \& Dodelson, S. 2002, ARA\&A, 40, 171

Jones, W. C., et al. 2005, ApJ, submitted [arXiv: astro-ph/0507494]

Kiss, C., Abraham, P., Klaas, U., Juvela, M., \& Lemke, D. 2001, A\&A, 379, 1161

Kogut, A., Spergel, D. N., Barnes, C., et al. 2003, ApJS, 148, 161

Komatsu, E., \& Seljak, U. 2002, MNRAS, 336, 1256

Kuo, C. L., Ade, P. A. R., Bock, J. J., et al. 2004, ApJ, 600, 32

Lagache, G., Haffner, L. M., Reynolds, R. J., \& Tufte, S. L. 2000, A\&A, 354, 247

Lagache, G., Puget, J.-L., \& Dole, H. 2005, ARA\&A, 43, 727

Ledlow, M. J., \& Owen, F. N. 1996, AJ, 112, 9

Leitch, E. M., Kovac, J. M., Halverson, N. W., et al. 2005, ApJ, 624, 10

Lewis, A., Challinor, A., \& Lasenby, A. 2000, ApJ, 538, 473, http: //camb.info

Liu, G.-C., da Silva, A., \& Aghanim, N. 2005, ApJ, 621, 15

Lo, K. Y., Chiueh, T. H., Martin, R. N., et al. 2001, in 20th Texas Symposium on relativistic astrophysics, AIP Conf. Proc., 586, 938

Mason, B. S., Pearson, T. J., Readhead, A. C. S., et al. 2003, ApJ, 591, 540

Matsuhara, H., Kawara, K., Sato, Y., et al. 2000, A\&A, 361, 407

Melchiorri, A., Choudhury, T. R., Serra, P., \& Ferrara, A. 2005, MNRAS, 364, 873

Miville-Deschênes, M.-A., Lagache, G., \& Puget, J.-L. 2002, A\&A, 393, 749

Montroy, T. E., Ade, P. A. R., Bock, J. J., et al. 2005, ApJ, submitted [arXiv:astro-ph/0507514]
Negrello, M., González-Nuevo, J., Magliocchetti, M., et al. 2005, MNRAS, 358, 869

Oh, S. P., Cooray, A., \& Kamionkowski, M. 2003, MNRAS, 342, L20

Pearson, T. J., Mason, B. S., Readhead, A. C. S., et al. 2003, ApJ, 591, 556

Piacentini, F., Ade, P., Bock, J., et al. 2005, ApJ, submitted [arXiv: astro-ph/0507507]

Readhead, A. C. S., Mason, B. S., Contaldi, C. R., et al. 2004a, ApJ, 609, 498 Readhead, A. C. S., Myers, S. T., Pearson, T. J., et al. 2004b, Science, 306, 836 Rebolo, R., Battye, R. A., Carreira, P., et al. 2004, MNRAS, 353, 747

Sheth, R. K., \& Tormen, G. 1999, MNRAS, 308, 119

Sokasian, A., Gawiser, E., \& Smoot, G. F. 2001, ApJ, 562, 88

Song, Y. S., Cooray, A., Knox, L., \& Zaldarriaga, M. 2003, ApJ, 590, 664

Sunyaev, R. A., \& Zel'dovich, Y. B. 1972, Comm. Astrophys. Space Phys., 4, 173

Sunyaev, R. A., \& Zel'dovich, Y. B. 1980, ARA\&A, 18, 537

Toffolatti, L., Argueso Gomez, F., De Zotti, G., et al. 1998, MNRAS, 297, 117

Toffolatti, L., Negrello, M., González-Nuevo, J., et al. 2005, A\&A, 438, 475

Tucci, M., Martínez-González, E., Vielva, P., \& Delabrouille, J. 2005, MNRAS, 360,935

White, M., \& Majumdar, S. 2004, ApJ, 602, 565

White, R. L., Becker, R. H., Gregg, M. D., et al. 1997, in 191st A\&AS Meeting, Bull. Amer. Astron. Soc., 29, 1373

Wu, J.-H. P. 2001, MNRAS, 327, 629 